\title{
Belle II physics prospects
}

\author{
Elisa Guido*广 \\ INFN Sezione di Torino, Via Pietro Giuria 1, I-10125, Torino, Italy \\ E-mail: elisa.guido@to.infn.it
}

The Belle II experiment at the SuperKEKB $B$-factory at KEK is expected to start collecting data at the beginning of 2018, opening a new era for collider physics. Complementary to the LHC experiments, Belle II is designed to explore the intensity frontier. Its target is to collect an integrated luminosity of $50 \mathrm{ab}^{-1}$ by 2024 , which is 50 times larger than the data sample recorded by the Belle experiment. The Belle II data will offer a unique possibility of searching for New Physics phenomena through precision study of rare and suppressed processes, as well as further revealing the nature of QCD in describing matter. The physics program of Belle II will be wide and only a selection of topics are highlighted in this paper: semileptonic and leptonic $B$ decays, electroweak penguins decays, time-dependent CP-violating decays, studies of mixing and rare decays in the charm sector, lepton flavour violation in $\tau$ decays, opportunities in bottomonium physics and searches for the dark sector.

The $15^{\text {th }}$ International Conference on Flavor Physics \& CP Violation 5-9 June 2017

Prague, Czech Republic

\footnotetext{
* Speaker.

${ }^{\dagger}$ On behalf of the Belle II Collaboration.
} 


\section{Introduction}

The BaBar and Belle experiments, namely the first generation of $e^{+} e^{-} B$-factories, left a solid heritage to future experiments and, in particular, to Belle II. The two experiments, in about a decade, collected data from $e^{+} e^{-}$collisions, mostly at the energy of the $\Upsilon(4 S)$ resonance, corresponding to an integrated luminosity of more than $1.5 \mathrm{ab}^{-1}$. The analysis of these samples allowed to discover the CP violation (CPV) and to confirm the Cabbibo-Kobayashi-Maskawa (CKM) description of flavour physics, but also to observe several new particles. In general, the two experiments carried out a diverse set of precision measurements of the Standard Model (SM), demonstrating it to be a healthy theory, but with some intriguing tensions. Currently, the most impressive anomalies have been observed in $B \rightarrow D^{(*)} \tau \nu_{\tau}$ and $B \rightarrow K^{(*)} \ell \ell$ decays and they eagerly wait for being confirmed with much larger data samples.

The Belle II experiment [1] at the SuperKEKB factory is expected to start taking data at the beginning of 2018 and to collect an integrated luminosity of $50 \mathrm{ab}^{-1}$ by 2024. Such a data sample will supersede that previously collected by the Belle experiment by a factor $\sim 50$ and will allow for precision studies of rare and suppressed processes, thus confirming the possible New Physics (NP) contribution to the current anomalies, finding others, and potentially understanding their correlations. Another crucial task for the Belle II physics program will be to further our understanding of the nature of QCD in describing matter.

Belle II will search for NP at the intensity frontier, thus acting a complementary role to the ATLAS and CMS experiments at the LHC that provide direct searches for NP at the energy frontier. There are two possible scenarios: if a NP signature were detected at the LHC, Belle II could determine its flavour structure, with unique access to the NP weak phases; alternatively, if no direct hint of NP were found at the LHC, indirect searches could be the right path to follow - for instance, through the search for $b \rightarrow s \gamma$ decays.

Belle II will also operate with healthy competition from the LHCb experiment. The advantages of Belle II with respect to a hadron-collider experiment are: it is a full solid-angle detector, working within the clean environment of $e^{+} e^{-}$collisions, allowing possibility of completely reconstructing the final states given the constrained kinematics. These features translate into the capability of studying missing-energy decays (such as $B \rightarrow D^{(*)} \tau v_{\tau}$ and $B \rightarrow \tau v_{\tau}$ ), performing inclusive measurements ( $b \rightarrow s \gamma, b \rightarrow s \ell \ell$ ), and efficiently reconstructing neutral particles, such as $\pi^{0}, \eta, \eta^{\prime}$ and $K_{L}^{0}$.

The expected physics program in the reach of Belle II is wide. It ranges over the following topics:

- $B$ physics: $\sim 1.1 \times 10^{9} B \bar{B}$ pairs for each collected $\mathrm{ab}^{-1}$ of data will be available for precision measurements of neutral $B$ mixing, penguin $B$ decays, and semileptonic and leptonic $B$ decays;

- Charm physics: Belle II will be a charm-factory as well, with $\sim 1.3 \times 10^{9} c \bar{c}$ pairs for each collected $\mathrm{ab}^{-1}$ of data, thus allowing for studies of mixing, $\mathrm{CPV}$, and rare decays in the charm sector;

- $\tau$ physics: with $\sim 0.9 \times 10^{9} \tau^{+} \tau^{-}$pairs for each collected $\mathrm{ab}^{-1}$ of data, NP contributions will be searched for in lepton flavour violating (LFV) $\tau$ decays; 
- Initial state radiation physics: Belle II will have access to a precise determination of the cross section for $e^{+} e^{-} \rightarrow$ light hadrons processes;

- Bottomonium and charmonium physics: it will be possible to further increase the current knowledge in the spectroscopy sector, as well as to discover new potential exotic states, mostly collecting data at the energy of the $\Upsilon(6 S)$ and $\Upsilon(3 S)$ resonances;

- Direct searches for NP at the MeV-GeV scale, for instance in the dark sector.

A selection of topics, among the many in the next generation physics program, is presented in this paper. More details about the physics reach of a $B$-factory in general, and of Belle II, in particular, can be found in [2] and [3], respectively.

Most of the expectations provided here are extrapolations from what were obtained by the Belle experiment, assuming similar efficiencies. Some differences in the Belle II detector with respect to Belle will have a relevant impact.

- Both Belle and Belle II exploit the beam energy asymmetry, which provides a boost to the $\Upsilon(4 S)$ system with respect to the laboratory frame such that the produced $B$ meson pair travels further along the beam axis; this allows for high-precision measurements of the decay time difference $(\Delta t)$ between the two $B$ mesons. In the case of Belle, whose main goal was the measurement of $\sin 2 \beta$, the energy asymmetry $(3.5 \mathrm{GeV}$ positrons (LER) on $8.0 \mathrm{GeV}$ electrons (HER)) corresponded to a boost of $\beta \gamma=0.425$. In the case of Belle II, the boost will be $\beta \gamma=0.28$ ( $4.0 \mathrm{GeV}$ positrons on $7.0 \mathrm{GeV}$ electrons), allowing the detector to have a better hermeticity. A higher hermeticity is a fundamental gain for the analyses of modes with neutrinos in the final state. The boost reduction of Belle II corresponds to a reduction in luminosity of $20 \%$ for the $J / \psi K_{S}, \phi K_{S}$ time-dependent measurements, while it represents a gain of $10 \%$ for the $B \rightarrow \tau \nu_{\tau}$ analysis.

- A smaller beam pipe radius (from $1.5 \mathrm{~cm}$ to $1.0 \mathrm{~cm}$ ) translates into an innermost silicon detector layer closer to the interaction point (from $2.0 \mathrm{~cm}$ to $1.3 \mathrm{~cm}$ ), as described in [1], and then into a significantly improved resolution in the $z$ direction.

- The outer radius of the Silicon Vertex Detector (SVD) has been significantly increased (from 8.8 to $14.0 \mathrm{~cm} \mathrm{[1])} \mathrm{and} \mathrm{will} \mathrm{allow} \mathrm{to} \mathrm{select} \mathrm{more} K_{S}^{0}$ candidates whose daughters have associated SVD hits, an important item for the time-dependent analyses using $K_{S}^{0}$ vertexing (e.g. $\left.B \rightarrow K_{S} \pi^{0} \gamma\right)$.

- Low momentum and SVD-only tracking improvements will allow for higher reconstruction efficiency of $D^{*}$ slow pions and better flavour tagging (slow pion category).

- A larger central drift chamber compared to Belle, with smaller cells, will allow for a better charged track reconstruction and $d E / d x$ measurement.

- Two Cherenkov detectors, the time-of-propagation detector in the barrel and the aerogel ring-imaging Cherenkov detector in the endcap, will provide particle identification (PID), with sensible improvements with respect to Belle [4], in particular for the $K / \pi$ separation; 
this will be a benefit for flavour tagging, rare charmless decays or $b \rightarrow s \gamma$ efficiencies and background rejection.

- Improvements to the outermost detector for the measurement of $K_{L}$ and muons (KLM) will result in higher $K_{L}$ veto efficiencies used in analyses looking at final states with some missing-energy (e.g. $B \rightarrow \tau \nu_{\tau}$ ).

\section{Semileptonic $B$ decays}

Semileptonic $B$ decays have been extensively used by BaBar and Belle to precisely determine the CKM parameters $\left|V_{\mathrm{ub}}\right|$ and $\left|V_{\mathrm{cb}}\right|$. At the end of the $B$-factories' era, we are left with some intriguing puzzles in this field. One of the most interesting open points consists in the 3- $\sigma$ tension between inclusive and exclusive measurements of $\left|V_{\mathrm{ub}}\right|$ and $\left|V_{\mathrm{cb}}\right|$, which is summarized in Fig. 1. The future challenge for experimentalists and theorists will be both to determine new techniques to understand this tension, profiting of a larger dataset, and to improve the theoretical predictions on $\left|V_{\mathrm{ub}}\right|$ and $\left|V_{\mathrm{cb}}\right|$. From the experimental point of view, exploiting the copious production of $\Lambda_{\mathrm{b}}$ in pp interactions, the LHCb experiment provided an important measurement of $\left|V_{\mathrm{ub}} / V_{\mathrm{cb}}\right|$ from barionic decays $\Lambda_{b} \rightarrow p \mu \nu$ [6], whose impact can be seen in Fig. 1. Relevant contributions are foreseen by the LHCb Collaboration even in the future, but Belle II will have access to more final states and will therefore be of crucial importance in the measurement of the sides of unitarity triangle and in understanding the nature of the current tension, with a unique access to the inclusive $\left|V_{\mathrm{ub}}\right|$ measurement. In particular, a precision of $\sim 2 \%(3 \%)$ is foreseen in the inclusive (exclusive) measurement of $\left|V_{\mathrm{ub}}\right|$ at Belle II.

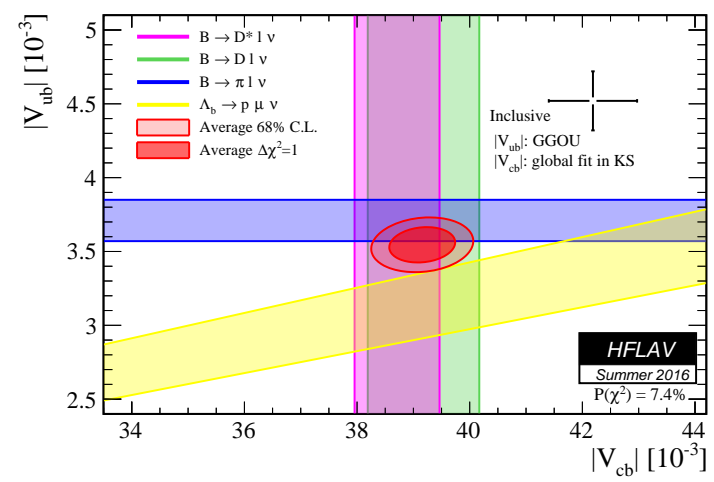

Figure 1: Combined exclusive measurements for $\left|V_{\mathrm{ub}}\right|$ and $\left|V_{\mathrm{cb}}\right|$, as from [5]. The combined values are $\left|V_{\mathrm{ub}}\right|=(3.55 \pm 0.12) \times 10^{-3}$ and $\left|V_{\mathrm{cb}}\right|=(39.16 \pm 0.58) \times 10^{-3}$. The inclusive value of $\left|V_{\mathrm{ub}}\right|$ from GGOU [7] and of $\left|V_{\mathrm{cb}}\right|$ from a global fit in the kinetic scheme [8] are also shown.

\section{3. $B \rightarrow \tau \nu_{\tau}$ and $B \rightarrow D^{(*)} \tau \nu_{\tau}$ decays}

Leptonic or semileptonic $B$ decay modes involving a $\tau$ lepton are very sensitive to NP, in the form of a charged Higgs contribution to the process. 
A 3.9- $\sigma$ deviation from the SM prediction has been observed in the $B \rightarrow D^{(*)} \tau v_{\tau}$ decays. The current experimental and theoretical status for the ratios of branching ratios (BRs): $R\left(D^{(*)}\right)=$ $\frac{\mathscr{B}\left(B \rightarrow \bar{D}^{(*)} \tau^{+} v_{\tau}\right)}{\mathscr{B}\left(B \rightarrow \bar{D}^{(*)} \ell^{+} v_{\ell}\right)}$ is summarized in Fig. 2. These ratios are predicted with high precision, of the order of 2-3\%. After collecting data for an integrated luminosity of $50 \mathrm{ab}^{-1}$, the Belle II experimental sensitivity is foreseen to be comparable to the current theoretical precision. This would translate in a potential 14- $\sigma$ exclusion of the SM prediction, if the measured central values remained the same as today. A larger data sample will also allow for performing additional interesting measurements, such as $D^{(*)}$ and $\tau$ polarization. The $\tau$ polarization has already been measured with the full Belle data sample [9], with the result $P(\tau)=-0.38 \pm 0.51$ (stat.) ${ }_{-0.16}^{+0.21}$ (syst.) being largely dominated by the statistical uncertainty.

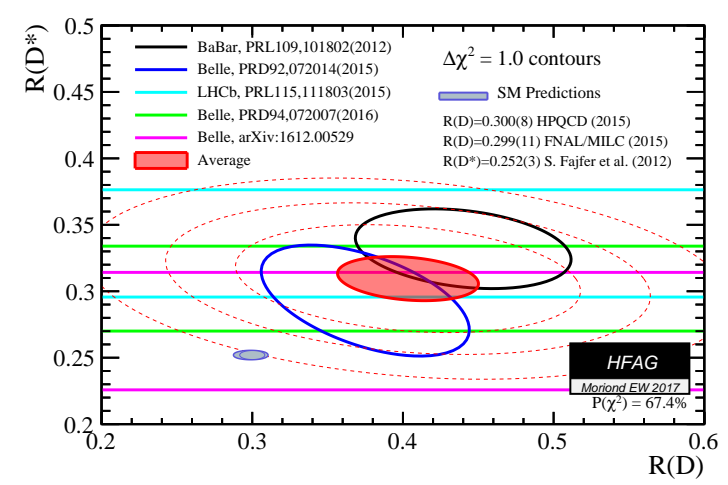

Figure 2: Averages of the ratios of BRs $R\left(D^{*}\right)=\frac{\mathscr{B}\left(B \rightarrow D^{*} \tau v_{\tau}\right)}{\mathscr{B}\left(B \rightarrow D^{*} \ell v_{\ell}\right)}=0.310 \pm 0.015$ (stat.) \pm 0.008 (syst.) and $R(D)=\frac{\mathscr{B}\left(B \rightarrow D \tau v_{\tau}\right)}{\mathscr{B}\left(B \rightarrow D \ell v_{\ell}\right)}=0.403 \pm 0.040$ (stat.) \pm 0.024 (syst.) from [5]. SM predictions are also shown. The input measurements and calculations are specified within the figure.

The decay $B \rightarrow \tau \nu_{\tau}$ is currently measured to have a BR of: $\mathscr{B}\left(B \rightarrow \tau \nu_{\tau}\right)=(1.09 \pm 0.24) \times$ $10^{-4}$ [10], where the average is obtained while considering results of analyses with both hadronic and semileptonic tags. The limited precision of the measurement does not allow for seeing any deviation with respect to the SM prediction. With $50 \mathrm{ab}^{-1}$ of Belle II data, the expected experimental precision will reach $\sim 6 \%$ (see Fig. 3), that will be comparable to the current theoretical precision on the predicted value.

\section{Electroweak penguin $B$ decays}

According to lepton universality, in the SM the ratio $\mathscr{R}_{K}=\frac{\mathscr{B}(B \rightarrow K \mu \mu)}{\mathscr{B}(B \rightarrow K e e)}$ is expected to be equal to 1 . The $\mathrm{LHCb}$ experiment has found a $2.6-\sigma$ deviation [12] from the SM expectation: $\mathscr{R}_{K}=$ $0.745_{-0.074}^{+0.090}$ (stat.) \pm 0.036 (syst.), in a limited region of low $q^{2}$, due to the difficulty of accessing $b \rightarrow$ see modes at high $q^{2}$. The measurements by BaBar [13] and Belle [14] are compatible with the SM prediction, but statistically limited. At Belle II the same detection efficiency is expected for both muons and electrons, thanks to an improved resolution in the ECL measurement of the electron momentum. The same detection efficiency is also expected at both low and high $q^{2}$, thus allowing to cross-check the measurement by LHCb and also to extend it to different $q^{2}$ regions. 


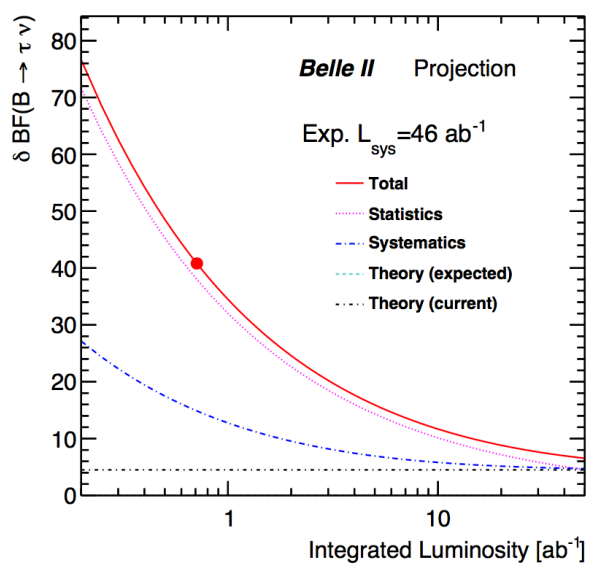

Figure 3: Projection of the expected experimental precision in the measurement of $\mathscr{B}\left(B \rightarrow \tau \nu_{\tau}\right)$ at Belle II, compared to the current theoretical uncertainty, as from [11]. The red point shows the current sensitivity obtained with the Belle dataset.

Similarly, LHCb has recently found a 2.1-2.5- $\sigma$ deviation from the SM expectation, depending on the $q^{2}$ region and on the prediction considered, in the $B \rightarrow K^{* 0} \ell \ell$ decays [15]. On the other hand, Belle has observed a 2.6- $\sigma$ tension with the SM in the angular distribution of the decays $B \rightarrow K^{*} \ell \ell[16]$.

A confirmation of these measurements by Belle II will be of vital importance on the path towards understanding whether these anomalies are real and, moreover, if they can be ascribed to a common source.

\section{Time-dependent $\mathrm{CP}$-violating $B$ decays}

The measurement of time-dependent CPV in $B$ decays was among the benchmark channels in the $B$-factories' program, giving access to the weak phase of the CKM matrix, through the interference between CPV in mixing and in decay. A precise determination of $\sin 2 \beta$ will still be important at Belle II, in order to check consistency of the unitarity triangle measurements with high-statistics data samples and also to search for new CP-violating phases in the decays $b \rightarrow s$ testing the SM prediction: $\sin 2 \beta(b \rightarrow s q \bar{q})=\sin 2 \beta(B \rightarrow J / \psi K)$. This kind of measurement involves basically all the aspects of the detector's performance: tracking efficiency, reconstruction of neutral particles, vertex reconstruction, PID, flavour tagging, as well as techniques for rejecting background events. In many of these aspects, significant improvements are expected at Belle II, for instance a better $\Delta t$ resolution ( $30 \%$ better than in Belle) and an enhanced flavour tagging efficiency.

Using the full dataset, Belle has obtained from $B \rightarrow c \bar{c} K^{0}$ decays: $\mathscr{S}=0.667 \pm 0.023$ (stat.) \pm 0.012 (syst.) and $\mathscr{A}=0.006 \pm 0.016$ (stat.) \pm 0.012 (syst.) [17], where $\mathscr{S}$ and $\mathscr{A}$ represent the CPV parameters. The expected Belle II sensitivity, using a data sample corresponding to an integrated luminosity of $50 \mathrm{ab}^{-1}$, will be: $\sigma_{\text {stat }}(\mathscr{S})=0.0027, \sigma_{\text {syst }}(\mathscr{S})=0.0044$, and $\sigma_{\text {stat }}(\mathscr{A})=0.0033$, $\sigma_{\text {syst }}(\mathscr{A})=0.0037$. The precision of the measurement will therefore be likely dominated by systematic uncertainties. 
The gluonic penguin $b \rightarrow s q \bar{q}$ decay modes (an example of Feynman diagram is shown in Fig. 4) have BRs foreseen with a high theoretical precision (of the order of $\sim 2 \%$ ) and all the current measurements - that are summarized in Fig. 5 - are consistent with what obtained on the $B \rightarrow J \psi K$ decay mode. Among these decays, there are some golden modes to search for additional CPviolating phases, given by particles beyond the SM entering the process loop: $B \rightarrow \phi K_{S}^{0}, B \rightarrow \eta^{\prime} K_{S}^{0}$ and $B \rightarrow K_{S}^{0} K_{S}^{0} K_{S}^{0}$. The expected precision for each of these decay modes when using $50 \mathrm{ab}^{-1}$ of data at Belle II are listed in Table 1, in comparison with the current results obtained by Belle. The foreseen uncertainties will be comparable to the current theoretical ones.

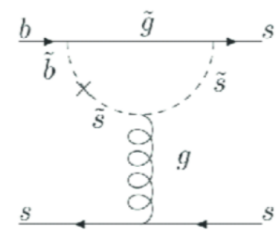

Figure 4: Example of Feynman diagram for a gluonic penguin $b \rightarrow s q \bar{q}$ decay.

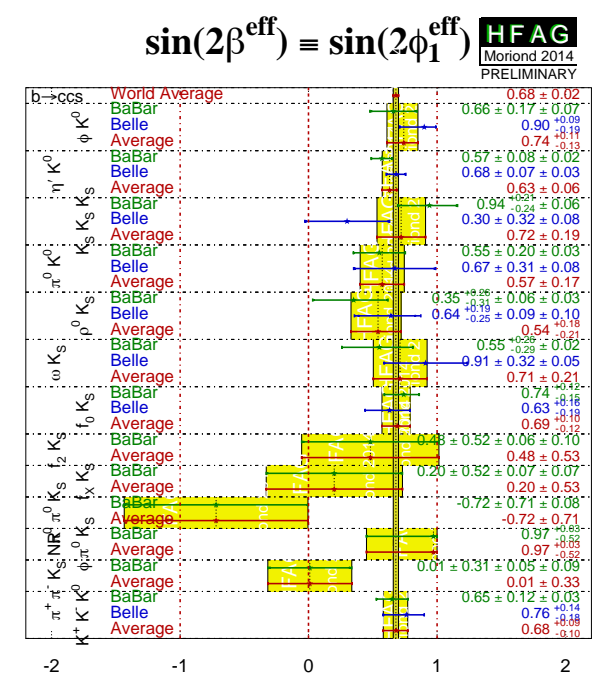

Figure 5: Compilation of results for $\sin \left(2 \beta^{\text {eff }}\right) \equiv \sin \left(2 \phi_{1}^{\text {eff }}\right)$ for several $b \rightarrow s q \bar{q}$ decay modes from [5].

\section{Charm physics}

After the discovery of $D^{0}-\bar{D}^{0}$ mixing by the $B$-factories, there is still a long wish list in the charm sector physics waiting for Belle II. It includes: improved measurements of the mixing parameters, search for direct and indirect CPV, search for rare decays. There will be several substantial improvements with respect to BaBar and Belle in the proper time resolution (it is expected to be $\sigma(t) \sim 0.14 \mathrm{ps}$, that is a factor 2 better than BaBar) and also in the flavour tagging (with the 
Table 1: Expected sensitivities on $\mathscr{S}$ and $\mathscr{A}$ using a data sample of $50 \mathrm{ab}^{-1}$ at Belle II, for three golden decay modes $b \rightarrow s q \bar{q}$, in comparison with the current Belle results.

\begin{tabular}{l|cc|cc}
\hline & \multicolumn{2}{|c|}{ Belle II } & \multicolumn{2}{c}{ Belle } \\
\hline Mode & $\sigma(\mathscr{S})$ & $\sigma(\mathscr{A})$ & $\sigma(\mathscr{S})$ & $\sigma(\mathscr{A})$ \\
\hline$B \rightarrow \phi K_{S}^{0}$ & 0.018 & 0.023 & 0.19 & 0.22 \\
$B \rightarrow \eta^{\prime} K_{S}^{0}$ & 0.011 & 0.009 & 0.08 & 0.06 \\
$B \rightarrow K_{S}^{0} K_{S}^{0} K_{S}^{0}$ & 0.033 & 0.021 & 0.33 & 0.21 \\
\hline
\end{tabular}

possibility of using new methods based on the study of the rest of the event). There will be also advantages for this kind of studied at an $e^{+} e^{-}$collider with respect to LHCb, basically given by the possibility of accessing the semileptonic decays, containing neutrinos in the final state, and also rare decays to neutral final states. An example of the latter is the flavour-changing neutral-current decay $D^{0} \rightarrow \gamma \gamma$, that is expected to have a BR of the order of $10^{-8}$. Belle recently looked for this decay, but did not find any statistically significant signal yield (see Fig. 6), thus setting an upper limit on this BR: $\mathscr{B}\left(D^{0} \rightarrow \gamma \gamma\right)<8.5 \times 10^{-7}$ at $90 \%$ of confidence level [18]. At Belle II, a data sample corresponding to an integrated luminosity of $50 \mathrm{ab}^{-1}$ will allow to measure a BR of the order of $10^{-7}-10^{-8}$ and therefore to possibly detect this decay.

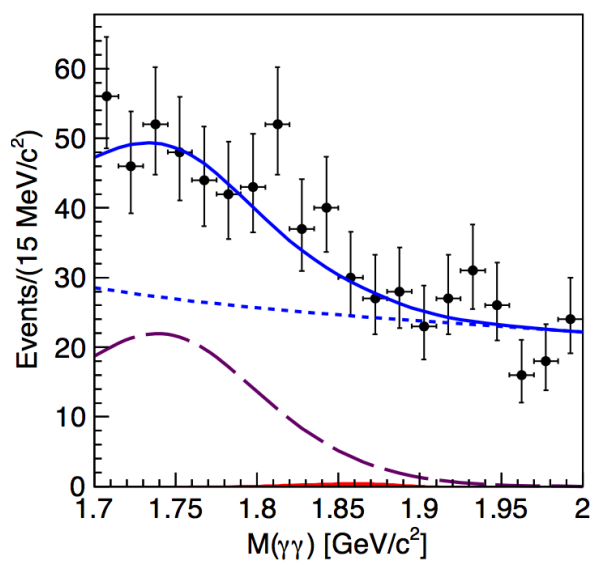

Figure 6: Projection of candidate events onto the $M(\gamma \gamma)$ distribution, as in [18]. Points with error bars are the data, blue solid curves are the results of the fit, blue dotted curves represent the combinatorial background, magenta dashed curves are the peaking background, and red filled histograms show the signal component.

\section{Lepton flavour violation in $\tau$ decays}

LFV $\tau$ decays are forbidden in the SM, but possibly enhanced in NP models, with BRs up to the order of $10^{-8}$. Belle II will uniquely access final states containing neutral particles (such as photons, $\pi^{0}, \eta, \eta^{\prime}$ ), with the competition from LHCb being possible only in a limited number of channels (namely, $\tau \rightarrow \mu \mu \mu$ decays). In these kinds of searches, the control of beam backgrounds 
will be crucial and will be precisely assessed only during data taking. Nevertheless, projecting the current upper limits to the expected integrated luminosities to be collected by Belle II, an improvement of 1-2 orders of magnitude is foreseen, as summarized in Fig. 7.

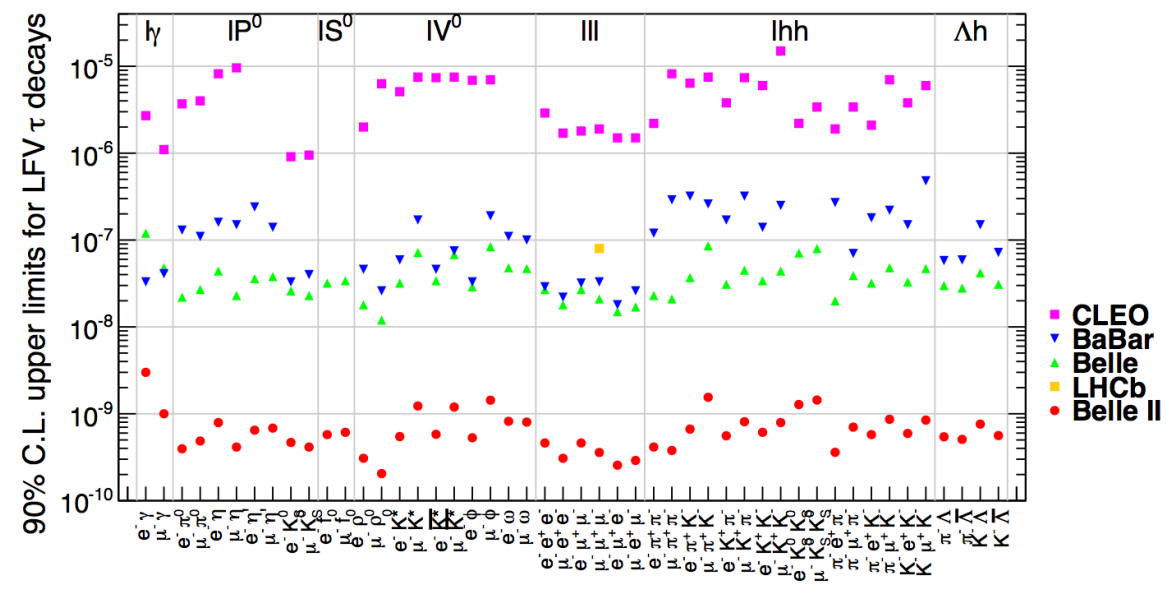

Figure 7: Upper limits on LFV $\tau$ decays (at 90\% of confidence level): extrapolations for Belle II (50 $\mathrm{ab}^{-1}$ ), as from [11], are compared to the current results from CLEO, BaBar, Belle and LHCb.

\section{Bottomonium physics}

The $B$-factories made great contributions to the knowledge of charmonium and bottomonium, discovering several unexpected quarkonium-like states, from $X(3872)$ to $Z_{b}(10610)$ and $Z_{b}(10650)$. Bottomonium physics at Belle II can achieve important results as well, through the study of some golden modes on a possibly unique data sample to be collected at the energy of the $\Upsilon(6 S)$ resonance. The first golden mode is the search for the $Z_{b}$ states from the decay $\Upsilon(6 S) \rightarrow Z_{b} \pi$, with $Z_{b} \rightarrow h_{b}(1 P) \pi$. Such a search has been attempted by Belle, using the data collected in a scan in the region of the $\Upsilon(6 S)$ [19], but the available statistics was not sufficient to measure the relative fraction of $Z_{b}(10610)$ and $Z_{b}(10650)$, as shown in Fig. 8. According to preliminary studies performed on Monte Carlo simulated samples, the separation between the two $Z_{b}$ states would be possible with a data sample corresponding to an integrated luminosity of $10 \mathrm{fb}^{-1}$. Also, $\eta$ transitions from the $\Upsilon(6 S)$ could be used to access the still missing states below $B \bar{B}$ threshold, such as the $\Upsilon(2 D)$ triplet, and to further study poorly known states, like the $\Upsilon(1 D)$ triplet. The transitions involving an $\eta$ meson have been of particular interest in the bottomonium physics program of the $B$-factories, since they imply a heavy-quark spin symmetry violation and provide an useful test bench of the QCD multipole expansion calculations. According to what has been seen from the analysis of the Belle data sample at the $\Upsilon(5 S)$ resonance [20], it is reasonable to expect cross sections below $1.5 \mathrm{pb}$ for $\eta$ transitions of the $\Upsilon(6 S)$ to lower bottomonia. From a preliminary estimate, it will be possible to measure these processes with a data sample corresponding to an integrated luminosity $\geq 50 \mathrm{fb}^{-1}$. 

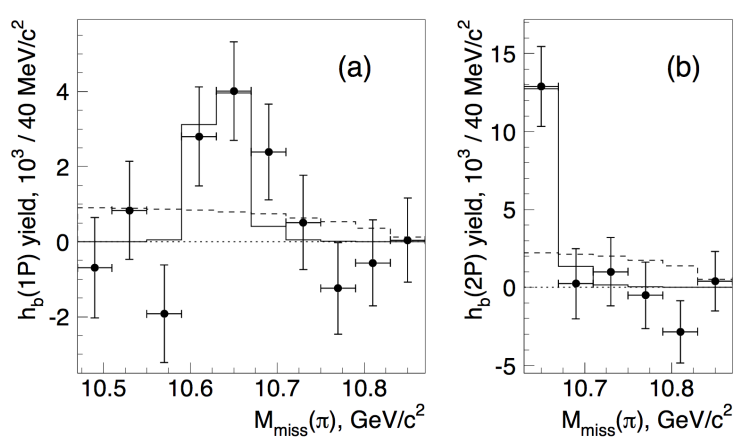

Figure 8: Pion missing mass distributions for $\Upsilon(6 S) \rightarrow h_{b}(1 P) \pi^{+} \pi^{-}$(a) and $\Upsilon(6 S) \rightarrow h_{b}(2 P) \pi^{+} \pi^{-}$(b) on the Belle data sample, as in [19]. Points represent data; the solid histogram represents the fit result with the $Z_{b}$ signal shape fixed from the $\Upsilon(5 S)$ analysis; the dashed histogram represents the result of the fit with a phase space distribution. The current statistics is not sufficient to disentangle the contribution from $Z_{b}(10610)$ and from $Z_{b}(10650)$

\section{Dark sector searches}

$B$-factories can also search for the hypothetical dark photon $\left(A^{\prime}\right)$ production in $e^{+} e^{-}$annihilations. The possibility of explaining the $(g-2)$ anomaly introducing exclusively a dark photon has been recently ruled out by searches for $A^{\prime}$ decaying into invisible final states by BaBar [21] and NA64 [22]. Nevertheless, the anomaly can still be interpreted in terms of a dark photon with the addition of some other NP contributions. Moreover, $A^{\prime}$ represents an important portal to light dark matter. A preliminary projection with $20 \mathrm{fb}^{-1}$ of data shows an impressive exclusion potential for Belle II on invisible decays of the dark photon. Such a data sample is likely to be collected even during the first phase of data taking, starting at the beginning of 2018. There is also another very challenging signature that is under study at Belle II, consisting in the search for $A^{\prime} \rightarrow \gamma+$ invisible. In this case, a special single photon trigger is required, besides an excellent tracking efficiency in order to reject events containing tracks. Clusters informations from the KLM will be used to reject events falling into the ECL gaps. Performance studies are on going and show promising results.

\section{Conclusions}

Belle II is ready to collect the legacy of the first generation of $B$-factories and to continue on the path set by them. Its main purpose will be searching for NP signatures at the intensity frontier, along with pursuing a deeper knowledge of the description of matter by QCD.

The physics program of Belle II will be thorough: a few highlights have been provided here, while many further ideas and developments are behind the corner, with the intriguing possibility of obtaining its first physics results already in 2018.

\section{References}

[1] T. Abe [Belle II Collaboration], "Belle II Technical Design Report”, arXiv:1011.0352 [physics.ins-det]. 
[2] T. Aushev et al., "Physics at Super B Factory", arXiv:1002.5012 [hep-ex].

[3] B2TiP report status is available at: https://confluence.desy.de/display/BI/B2TiP+ReportStatus.

[4] I. Adachi et al., "sBelle Design Group", sBelle Design Study Report, arXiv:0810.4084 [hep-ex].

[5] Y. Amhis et al., "Averages of $b$-hadron, $c$-hadron, and $\tau$-lepton properties as of summer 2016," arXiv:1612.07233 [hep-ex].

[6] R. Aaij et al. [LHCb Collaboration], "Determination of the quark coupling strength $\left|V_{\mathrm{ub}}\right|$ using baryonic decays," Nature Phys. 11 (2015) 743 [arXiv:1504.01568[hep-ph]].

[7] P. Gambino, P. Giordano, G. Ossola and N. Uraltsev, "Inclusive semileptonic B decays and the determination of $\left|V_{\mathrm{ub}}\right|$, , JHEP 0710 (2007) 058 [arXiv:0707.2493 [hep-ph]].

[8] P. Gambino and C. Schwanda, "Inclusive semileptonic fits, heavy quark masses, and $V_{\mathrm{cb}}$," Phys. Rev. D 89 (2014) no.1, 014022 [arXiv:1307.4551 [hep-ph]].

[9] S. Hirose et al. [Belle Collaboration], "Measurement of the $\tau$ lepton polarization and $R\left(D^{*}\right)$ in the decay $\bar{B} \rightarrow D^{*} \tau^{-} \bar{v}_{\tau}$," Phys. Rev. Lett. 118 (2017) no.21, 211801 [arXiv:1612.00529 [hep-ex]].

[10] C. Patrignani et al. [Particle Data Group], Chin. Phys. C 40, 100001 (2016).

[11] B. Golob, K. Trabelsi and P. Urquijo, Impact of Belle II on flavour physics, BELLE2-NOTE-PH-2015-002.

[12] R. Aaij et al. [LHCb Collaboration], "Test of lepton universality using $B^{+} \rightarrow K^{+} \ell^{+} \ell^{-}$decays," Phys. Rev. Lett. 113 (2014) 151601 [arXiv:1406.6482 [hep-ex]].

[13] J. P. Lees et al. [BaBar Collaboration], "Measurement of Branching Fractions and Rate Asymmetries in the Rare Decays $B \rightarrow K^{(*)} l^{+} l^{-}$," Phys. Rev. D 86 (2012) 032012 [arXiv:1204.3933 [hep-ex]].

[14] J.-T. Wei et al. [Belle Collaboration], "Measurement of the Differential Branching Fraction and Forward-Backword Asymmetry for $B \rightarrow K^{(*)} \ell^{+} \ell^{-}$," Phys. Rev. Lett. 103 (2009) 171801 [arXiv:0904.0770 [hep-ex]].

[15] R. Aaij et al. [LHCb Collaboration], "Test of lepton universality with $B^{0} \rightarrow K^{* 0} \ell^{+} \ell^{-}$decays," JHEP 1708 (2017) 055 [arXiv: 1705.05802 [hep-ex]].

[16] S. Wehle et al. [Belle Collaboration], "Lepton-Flavor-Dependent Angular Analysis of $B \rightarrow K^{*} \ell^{+} \ell^{-}$," Phys. Rev. Lett. 118 (2017) no.11, 111801 [arXiv:1612.05014 [hep-ex]].

[17] I. Adachi et al. [Belle Collaboration], "Precise measurement of the CP violation parameter $\sin 2 \phi_{1}$ in $B^{0} \rightarrow(c \bar{c}) K_{0}$ decays," Phys. Rev. Lett. 108 (2012) 171802 [arXiv:1201.4643 [hep-ex]].

[18] N. K. Nisar et al. [Belle Collaboration], "Search for the rare decay $D^{0} \rightarrow \gamma \gamma$ at Belle," Phys. Rev. D 93 (2016) no.5, 051102 [arXiv:1512.02992 [hep-ex]].

[19] A. Abdesselam et al. [Belle Collaboration], "Energy scan of the $e^{+} e^{-} \rightarrow h_{b}(n P) \pi^{+} \pi^{-}(n=1,2)$ cross sections and evidence for $\Upsilon(11020)$ decays into charged bottomonium-like states," Phys. Rev. Lett. 117 (2016) no.14, 142001 [arXiv:1508.06562 [hep-ex]].

[20] Belle Collaboration, Preliminary results shown by P. Krokovny at the conference LaThuile 2012 and by U. Tamponi at the conference DIS 2014.

[21] J. P. Lees et al. [BaBar Collaboration], "Search for invisible decays of a dark photon produced in $e^{+} e^{-}$collisions at BaBar," arXiv:1702.03327 [hep-ex].

[22] D. Banerjee et al. [NA64 Collaboration], "Search for invisible decays of sub-GeV dark photons in missing-energy events at the CERN SPS," Phys. Rev. Lett. 118 (2017) no.1, 011802 [arXiv: 1610.02988 [hep-ex]]. 